DOE/ER/14213--1

DE93 014098

\title{
RADIATIVE PROPERTIES OF STRONGLY \\ MAGNETIZED PLASMAS
}

Progress Report

September 1991 - December 1992

J. C. Weisheit

Department of Space Physics and Astronomy

Rice University

Houston, Texas 77251-1892

December 1992

\section{PREPARED FOR THE U.S. DEPARTMENT OF ENERGY UNDER GRANT NUMBER DE-FG05-01ER14213}

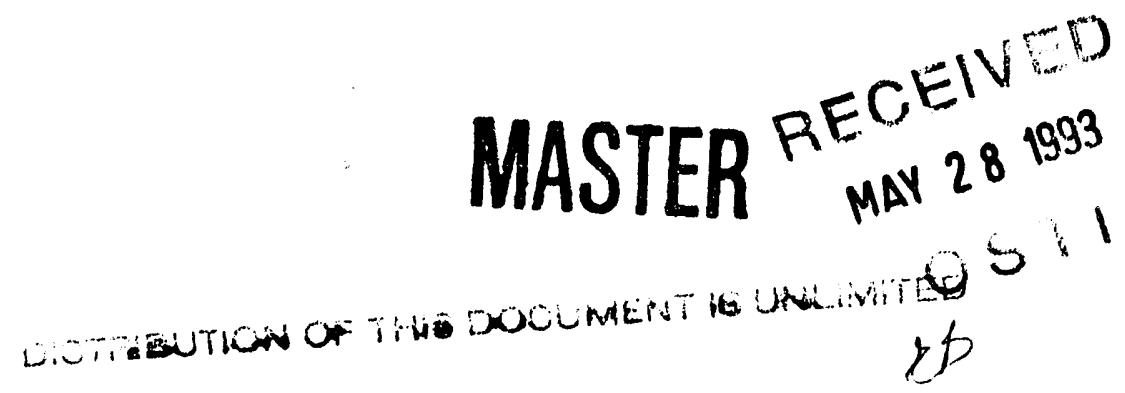




\section{PROGRESS IN THE PRESENT BUDGET PERIOD}

The subject of atomic properties in the presence of very strong magnetic fields is experiencing a new wave of interest, especially insofar as non-hydrogenic systems are concerned, and we believe the research summarized here is on the crest of that wave. Only recently there have appeared a major review of Thomas-Fermi theory [Spruch, L. 1991, Rev. Mod. Phys. 63, 151]; a new set of fundamental theorems pertaining to the Hamiltonian of a (ThomasFermi) atom in a strong field [ Lieb E.H., Solovej J.P., Yngvason J., Phys. Rev. Lett. 69, 749 (1992)]; and the first numerical, Hartree-Fock (HF) results for multi-electron atoms in strong $B$ fields, but obtained under the restrictive assumption that the $\varrho$ - and $z$-dependence of individual orbitals is completely separable [ Miller M.C., Neuhauser D. Mon. Not. R. astr. Soc., 253, 107 (1991)].

\section{THEORETICAL ANALYSIS}

Multi-electron atoms in a strong magnetic field have an elongated form, since the field "compresses" all transverse dimensions. Nevertheless, many previous studies of such atoms assumed a spherically symmetric shape, thereby reducing the problem to one dimension. In our work the more natural cylindrical symmetry is adopted, in which the charge density $n \equiv n(\varrho, z)$ factorizes according to

$$
n(\varrho, z)=n_{\varrho}(\varrho) n_{z}(\varrho, z)
$$

This more general ansatz differs from the complete factorization [where $n_{z}=n_{z}(z)$ ] which has been assumed elsewhere. The price for this generalization is considerable complication of the basic equation derived by Kadomtsev for a one-dimensional Thomas-Fermi model and solved by him for the spherically symmetric case. With our ansatz the nonlinear differential equation for the factor $n_{z}$ of the charge density becomes

$$
\frac{\partial^{2} n_{z}^{2}}{\partial \tilde{z}^{2}}+\frac{\partial^{2} n_{z}^{2}}{\partial \tilde{\varrho}^{2}}+C(\tilde{\varrho}) \frac{\partial n_{z}^{2}}{\partial \tilde{\varrho}}=-A(\tilde{\varrho}) n_{z}^{2}+D(\tilde{\varrho}) n_{z}
$$

where $\hat{\varrho}=\sqrt{2 / B}$ is the quantum cyclotron radius, which serves as a natural unit of length in the problem, $\tilde{\varrho}=\varrho / \hat{\varrho}, \quad \tilde{z}=z / \hat{\varrho}, \quad$ and where

$$
A(\tilde{\varrho})=\left(\frac{\partial^{2} n_{\ell}^{2}}{\partial \tilde{\varrho}^{2}}+\frac{1}{\tilde{\varrho}} \frac{\partial n_{\varrho}^{2}}{\partial \tilde{\varrho}}\right) / n_{\ell}^{2}, C(\tilde{\varrho})=\frac{1}{\tilde{\varrho}}+\frac{2}{n_{\ell}^{2}} \frac{\partial n_{\ell}^{2}}{\partial \tilde{\varrho}}, D(\tilde{\varrho})=2 /\left(\pi^{3} \hat{\varrho}^{2} n_{\varrho}\right) .
$$

A few authors have assumed that the charge density has the shape of coaxial cylinders, corresponding to different values of the orbital angular momentum projection. We found that $n_{e}(\varrho)$, which can be calculated using Landau wavefunctions, is an almost uniform function of the radius, with a sharp drop at the edge. The explicit form of this function allows us to calculate all the coefficients in Eq. 2, so all we have to do is to solve it.

Equation 2 is a non-linear, elliptical equation and does not have an analytical solution in its general form; but, there are simple analytical solutions which we obtained with additional 
assumptions. The most crude assumption, that of full factorization of $n(\varrho, z)$, gives us a solution which resembles the trial exponential function frequently used by others without sufficient foundation. The next assumption, of " $z$-derivatives domination", leads to a shape predicted before, from semi-qualitative considerations. Both solutions have the required normalization, which is not trivial for the non-linear equation, and both yield an elongated shape of an atom with length and width being "compressed" with increasing magnetic field (as expected).

Neither of these assumptions is valid near the nucleus. In its vicinity we should have nearly a pure Coulomb solution. The easiest way to correct the previous results is just to add the Coulomb term to the solutions. It is preferable, though, to solve the full Eq. (2) without any further simplifications. Ultimately, a numerical attack becomes necessary.

\section{NUMERICAL ANALYSIS}

Equation (2) represents a combined Dirichlet-Neuman problem on a two-dimensional domain. Due to the symmetry of the problem, it is sufficient to consider only one quadrant with proper boundary conditions. We require a Dirichlet condition at one border and Neuman conditions at two others. Since the unknown function $n_{z}$ is being multiplied by $n_{\ell}$ to give the charge density $n$, and $n_{\rho}$ is sharply falling at the edge of the atom, we choose not to specify Dirichlet conditions at the remaining border; instead we demand the "cylinderwise" normalization condition adequate to the physical nature of Landau levels:

$$
\int_{V} n(\varrho, z) \mathrm{d} V=Z \int_{-\infty}^{+\infty} n_{z}(\varrho, z) \mathrm{d} z=Z
$$

This normalization means that the radial distribution of the charge density is as prescribed by Landau wavefunctions and so, actually, $n_{z}(\varrho, z)$ just determines the $z$-distribution in a given Landau "cylinder". From the mathematical point of view such a substitution, for the fourth boundary condition, is not conventional but is formally allowed for a square domain, since it gives the same number of equations for calculating values of the function at the nodes of a grid. Finally, to ensure proper Coulomb asymptotics, we specify a value of $n_{z}$ in the node nearest the nucleus. At this node we can consider the nucleus as an unscreened point charge.

Very few computational methods seem promising. The simplest numerical method which might be used is a finite difference scheme. If we chose a square $k \times k$ grid that covers all the domain (the whole coordinate plane or only a quadrant in the case of axial symmetry) and try to calculate the val'ves of the electron density $n_{z}$ at the nodes, we will have a set of $(k+1) \times(k+1)$ unknown values $\left(n_{z}\right)_{i, j}$, where $0 \leq i, j \leq k$. To determine these values one may impose $4 k$ boundary conditions of Dirichlet, Neuman, and "normalization" type and write $(k-1) \times(k-1)$ equations (one for each internal node). Unfortunately, there is a feature that sufficiently complicates the solution of the system of the equations that results. It comes from the nonlinearity of the Thomas-Fermi equation itself, but if we neglect the nonlinear term we lose the essential effect.

A nonlinear differential equation of the second order can still be solved by finite difference methods if one performs iterations, using Pickard or Newton-Raphston techniques. To avoid 
this complicated procedure we instead tried to solve the equation for one point after another, "step by step", propagating the solution outward from the nucleus. There are certain advantages to this approach but they are outweighted by two important disadvantages. The first negative feature is that only a cruder normalization condition can be imposed (only overall normalization, rather than "cylinderwise"). The second and more serious negative feature is that the numerical solution usually exhibits exponential growth. We found that this instability originates in the method itself: if for any reason the second derivative with respect to $\varrho$ will be slightly smaller than it should be then, since the value of Laplacian is fixed, the other second order derivative becomes slightly larger. At the next step this error enhances itself. Due to the nature of the method, the solution does not "feel" the boundary condition "ahead" and fails to match it. This feature makes the method us less here because the required number of steps is large.

Our present approach involves the finite element method. It also presumes solution of a large system of linear equations like the finite difference method. That makes it necessary to perform iterations to solve the original nonlinear equation. On the other hand, unlike the finite difference method, it can be adapted to specific features of the problem. It is possible, for example, to make the grid denser in the most critical points and sparser in the others. In particular, variational and Galerkin approaches can be pursued in the finite element method. A variational solution should not exhibit any instabilities and unexpected growth. Another feature is its natural connection with the concept of energy, since among all the possible solutions of the Kadomtsev equation only one with the lowest energy will be correct. The usual drawback is the difficulty one has to choose a functional for a non-linear problem.

At present, the Galerkin scheme is being used, in a version of the finite element method that can be applied together with an iteration procedure to handle the nonlinearity of the equation. Finite elements in the form of triangles have been chosen. Our computer code is now in the debug stage.

\section{PLAN FOR THE NEXT BUDGET PERIOD (AND BEYOND)}

The numerical algorithms under development here evidently will provide the quantitative means of determining just how important is the restriction of complete $\varrho$ and $z$ factorization, and just how low in field intensity $B$ one can safely employ it. Therefore, we are pursuing "Part B" of the original proposal in greater depth than outlined therein. We still plan to use our axial Thomas-Fermi charge density to make a local exchange approximation in a non-spherical, self-consistent field model, but now we also plan to study carefully other fundamental issues, such as:

- a comparison of ground-state energies determined in different approaches

- elastic scattering (magnetic Thomas-Fermi) form factors for determining magnetized plasma resistivities 
- the effect of intense field $B$ on the collapse conditions for magnetic white dwarf stars [themselves treated as $N\left(\sim 10^{50}\right)$-electron Thomas-Fermi "atoms"].

My postdoctoral research associate, Dr. Gene Lief, will be putting most of his efforts on this problem area.

The non-trivial numerical challenge posed by the 2-dimensional generalization of Kadomsev's equation we derived has so far precluded much serious work on "Part A" of the proposal - the lineshape problem. However, this will change next month, as I begin to explore the application of my new, efficient computer routine for two-component plasma structure factors to the question of time-dependent microfields and the coupling of Stark and Doppler broadening effects when $\boldsymbol{B}$ is intense.

Somewhat serendipitously, this work by Lief and me has come to the attention of three very different physics research programs recieving Dept. of Energy support. In the long term, this project could be strongly influenced by one or more of them. First (as a member of the external advisory panel for Los Alamos X Division) I learned of the LANL "Trailmaster" program that investigates physics of magnetically driven foil implosions. Radiative cooling is important here, but LANL codes presently must ignore any and all effects of the megagauss fields on radiative opacities. This program may yield the best comprehensive test of a (magnetic) opacity scheme. Second, Lief met C. Froese-Fischer at a spectroscopy conference last September, and discussions have ensued as to how her elaborate HF codes might be extended to include strong B fields. Third, during our recent Contractors' Workshop, I met D. Hammer, the director of Cornell's Plasma Studies Lab. It seems possible to generate megagauss fields in his pinch plasma experiments, for which moderate-resolution $\mathrm{X}$-ray spectroscopic data are routinely taken. Such data, coupled with elaborate non-LTE plasma modelling codes, may reveal important B-field effects on the rates of specific atomic processes. 

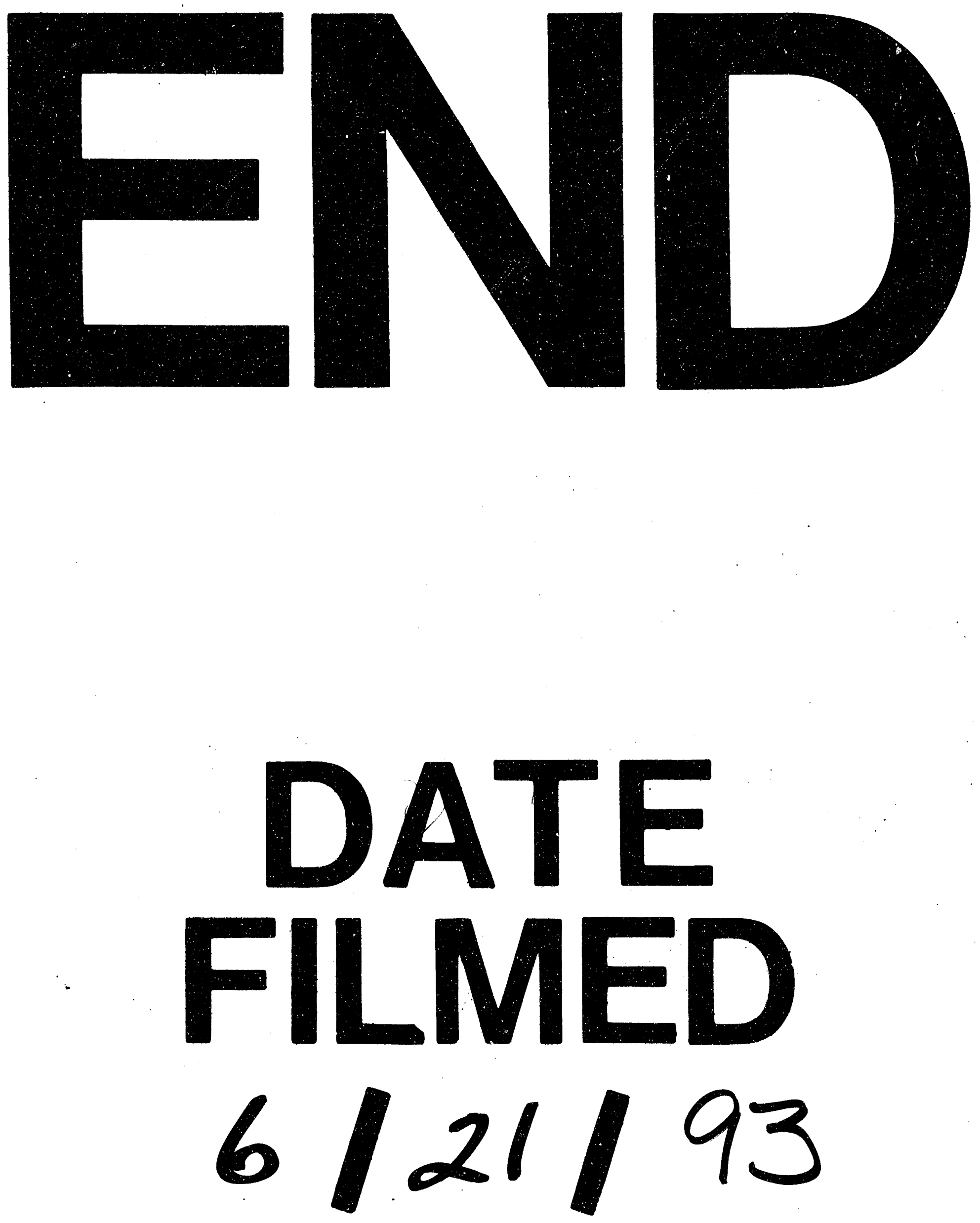
